\title{
AUTOMATIC DETECTION OF DUST AND SCRATCHES IN SILVER HALIDE FILM USING POLARIZED DARK-FIELD ILLUMINATION
}

\author{
Dominic Rüfenacht ${ }^{1}$, Giorgio Trumpy ${ }^{2}$, Rudolf Gschwind ${ }^{2}$, and Sabine Süsstrunk ${ }^{1}$ \\ ${ }^{1}$ School of Computer and Communication Sciences, EPFL, Switzerland \\ ${ }^{2}$ Imaging and Media Lab, University of Basel, Switzerland
}

\begin{abstract}
We present a method to automatically detect dust and scratches on photographic material, in particular silver halide film, where traditional methods for detecting and removing defects fail. The film is digitized using a novel setup involving crosspolarization and dark-field illumination in a cardinal light configuration, which compresses the signal and highlights the parts that are due to defects in the film. Applying a principal component analysis (PCA) on the four cardinal images allows us to further separate the signal part of the film from the defects. Information from all four principal components is combined to produce a surface defect mask, which can be used as input to inpainting methods to remove the defects. Our method is able to detect most of the dust and scratches while keeping false-detections low.
\end{abstract}

Index Terms - Dust and scratch detection, Principal Component Analysis, Adaptive thresholding.

\section{INTRODUCTION}

Silver-halide based photographic material is not permanent, which is why there is a great interest in digitizing these visual documents. Manual restoration of dust and scratches present on film is both time-consuming and expensive. Also, due to the vast number of films in archives that need to be restored, an automatic framework is needed. We propose a method to automatically detect surface defects (dust and scratches) on any kind of transparent photographic material, in particular on black and white film. This is achieved by a, for this application, novel physical setup that uses cross-polarization and dark-field illumination [1], combined with subsequent image processing to create the defect mask.

Several methods have been proposed to detect and remove surface defects on film. On the acquisition side, the most effective solution is called infrared cleaning [2]. This method takes a "normal" visible image using white light, and one in the Near-Infrared (NIR) using an NIR light source. The color dyes present in color film are transparent to NIR, and hence defects are visible in the NIR and can subsequently be

This work is supported by the Swiss National Science Foundation under grant number 200021-130261. removed. This method works well for color transparencies with small defects, but does not work for black and white (silver halide) film, since the silver grains are not transparent in the NIR.

WetGate [3] is an older method where at the time of exposure, the film is coated with a liquid that has a suitable refractive index to cover scratches on the film surface. It involves the use of toxic chemicals, and dust and other particles are not removed. The film also needs to be cleaned afterwards.

In [4], a semi-automatic framework for detecting and removing dust and scratches from any type of film is proposed by using diffuse light to illuminate the film. Their dirt and scratch correction option (D/SCO) generates a map of surface and deeper defects depending on user-defined, imagedependent parameters.

Various methods have been proposed to restore film digitized by conventional scanning. In the case of moving image film, several methods exploit the temporal correlation between consecutive frames to detect (and remove) defects [5-7]. Spatio-temporal approaches tend to have problems with movements in the scene, as the movement needs to be compensated for. Methods using single still images simplify the problem by focusing on specific defect orientations such as vertical scratches [8], and therefore do not generalize well.

Our method combines a novel film acquisition setup with image processing, and can be used to detect defects in both still and moving images. In particular, our fully automatic method works for the detection of both dust and scratches on black and white film, irrespective of the defect size and orientation. We acquire four cardinal images using a combination of cross-polarization and dark-field illumination, where the only difference is the position of the light source with respect to the film. In these cardinal images, defects are highlighted, and the content of the film is suppressed. We use these images to build both a coarse and a refined defect map, which can be used for subsequent image restoration techniques.

The rest of the paper is organized as follows. In Section 2, we explain the experimental setup. We then present our workflow to automatically detect defects in Section 3. Qualitative and quantitative results are presented in Section 4, and Section 5 concludes the article. 


\section{EXPERIMENTAL SETUP}

We use the setup first presented by Trumpy et al. [9], and acquire a direct light image and images in which the defects are emphasized. Fig. 1 shows a schematic of the setup.

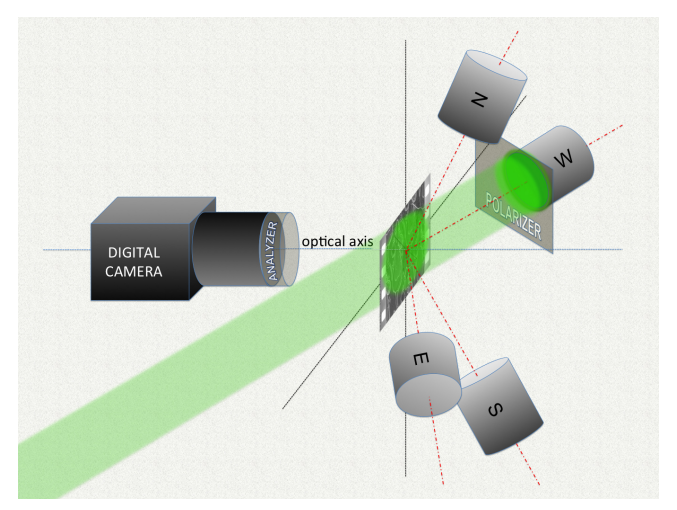

Fig. 1. Setup to capture the four cardinal images.

We use cross-polarization by using two orthogonally oriented polarizing filters (one on the light source, one on the lens of the camera). While the silver grains of the film do not perturb the polarization of light, the surface defects do. Therefore, the signal related to the silver emulsion is suppressed by the orthogonally polarized filter on the lens, while the signal related to the defects is maintained. The dark-field method suppresses the signal related to the silver emulsion by excluding the non-scattered beam from the image. The silver grains in the emulsion of the film are randomly dispersed, and their interaction with light is isotropic. The scattered light therefore produces a similar signal irrespective of the position of the light source. Dust and scratches on the film surface, on the other hand, produce reflections and shadows, which strongly depend on the light direction. We therefore use a cardinal setup with four light directions (i.e., North, South, East, and West), where the angle $\theta$ between the direction of the illumination and the optical axis (see Figure 1) is the same. In our setup, we use $\theta=45^{\circ}$. We also take one direct light image without polarization, where the light source is placed in the optical axis.

\subsection{Image Model}

In the following, we denote $a_{i j}$ the value of a pixel at location $(i, j)$, where $i \in[1, m]$ and $j \in[1, n]$, of a matrix $\boldsymbol{A}$ of $m$ rows and $n$ columns. We acquire four cardinal images $\boldsymbol{C}^{(k)}, k \in\{1,2,3,4\}$ and a direct light image $\boldsymbol{L}$. The direct light image pixel values are modelled as follows:

$$
l_{i j}=\left(1-d_{i j}\right) s_{i j}+d_{i j} w_{i j}+\sigma_{i j},
$$

where $s_{i j}$ is the actual signal part in the film, $d_{i j}$ is a pixel of the binary mask of defects $\boldsymbol{D}$ (dust and scratches) of weight $w_{i j}$, and $\sigma$ is camera noise.The pixel values $c_{i j}^{(k)}$ of the four cardinal images $\boldsymbol{C}^{(k)}$ are modelled in a similar way:

$$
c_{i j}^{(k)}=\left(\alpha \cdot \zeta^{(k)}\right)\left(1-d_{i j}\right) s_{i j}+\kappa d_{i j} w_{i j}+\gamma \sigma_{i j},
$$

where $\alpha \ll 1, \kappa \geq 1$, and $\gamma>1$ are attenuation and amplification factors due to the cross-polarized dark field illumination. $\zeta^{(k)}<1$ accounts for shadows cast by the defects on the film surface, and is the main difference between the four cardinal images.

\section{DUST AND SCRATCH DETECTION ALGORITHM}

Fig. 2 gives an overview of the proposed defect detection pipeline.

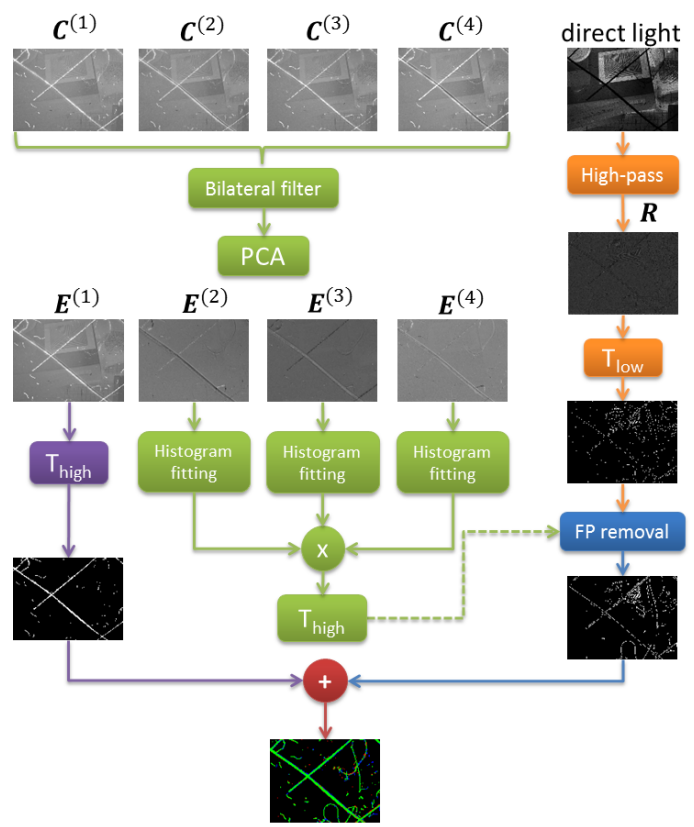

Fig. 2. Proposed pipeline for dust and scratch detection.

We start by registering all images using SIFT [10] with RANSAC [11]. We then apply a bilateral filter [12] on the four cardinal images $\boldsymbol{C}^{(k)}$ to remove the noise $\sigma$, while at the same time conserving the (larger) defect parts. The filtered $\boldsymbol{C}^{(k)}$ are the input to a principal component analysis (PCA). We compute first a coarse defect map by only considering the first principal component $\boldsymbol{E}^{(1)}$. While we are able to detect most major defects, we miss thin defects that are below the signal to noise ratio (SNR) of the cardinal images. We thus propose a refinement step where we use the (less noisy) direct light image combined with the second to fourth principal components to add thin defects.

\subsection{Coarse Defect Map}

The output of the PCA on the four cardinal images $\left(\boldsymbol{C}^{(1)}-\boldsymbol{C}^{(4)}\right)$ are the four principal components $\left(\boldsymbol{E}^{(1)}-\boldsymbol{E}^{(4)}\right)$, where $\boldsymbol{E}^{(1)}$ 
expresses the spatial variability of the image, and $\left(\boldsymbol{E}^{(2)}-\boldsymbol{E}^{(4)}\right)$ emphasize the differences due to the defects on the film surface (see Fig. 2). Big defects are bright in all four images and therefore do not change, and hence are contained in the first principal component. Fig. 3 shows an example of $\boldsymbol{E}^{(1)}$, as well as its corresponding histogram.

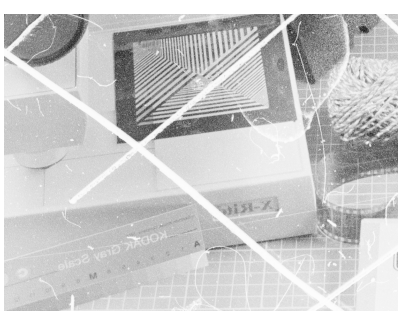

(a) Direct light image $\boldsymbol{L}$.

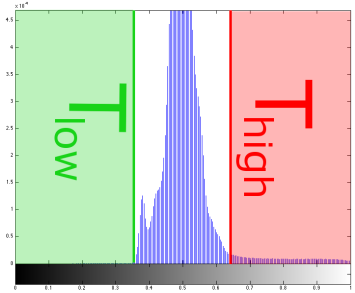

(c) Histogram of $\boldsymbol{E}^{(1)}$

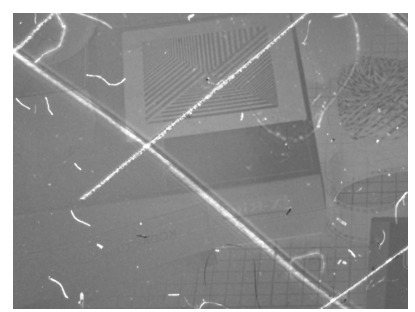

(b) $\boldsymbol{E}^{(1)}$

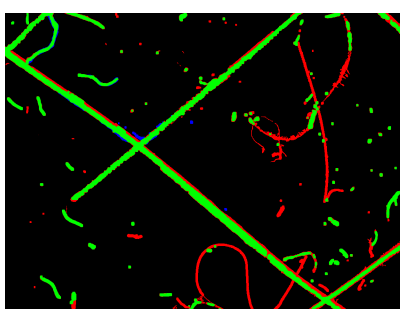

(d) Coarse defect map $D^{\text {coarse }}$.
Fig. 3. Example of (a) direct light image $\boldsymbol{L}$, (b) first principal component of the cardinal images, (c) the histogram of $\boldsymbol{E}^{(1)}$, and (d) the coarse defect map. True positives are green, false positives blue, false negatives red, and true negatives black.

The film signal values are compressed, while most defects are highlighted in the bright parts. To compute the defect map, we threshold the image based on the histogram (see Fig. 3(c)). We set $T_{\text {high }}\left(T_{\text {low }}\right)$ to be the largest (lowest) location where the absolute difference between consecutive histogram bins is larger than an image-size dependant value. The coarse defect map is computed as follows:

$$
d_{i j}^{\text {coarse }}= \begin{cases}1 & \text { if } e_{i j}^{(1)}>T_{\text {high }} \\ 0 & \text { otherwise }\end{cases}
$$

Fig. 3(d) shows a (color-coded) coarse defect map. While most of the major defects are correctly detected, as well as almost no false positives, thin defects are missing.

\subsection{Thin Defect Map}

Due to the considerable amount of noise introduced by the dark field illumination and subsequent long exposure, thin defects that are below the SNR will not be completely detected. Many defects have a rather elongated, continuous structure, which is partially lost in the cardinal images. They are, however, still present in the much less noisy direct light image $\boldsymbol{L}$. In the end, we want to detect (and remove) the defects of the direct light image, and hence we also need to detect those thin defects. Finding a threshold that separates the defects from the image parts in the direct light image $\boldsymbol{L}$ is difficult since bright parts in the film are hardly distinguishable from scratches. By combining the direct light image with the information contained in the second to fourth principal components, we can derive a detailed defect map $D^{\text {thin }}$ to augment our previously calculated coarse defect map $D^{\text {coarse }}$.

We first apply the function $r_{i j}=\log \left(l_{i j}\right)-\log \left(g\left(l_{i j}\right)\right)$ to the direct light image $\boldsymbol{L}$ in order to extract the high-frequency components of $\boldsymbol{L} . g(x)$ is a low-pass Gaussian filter. We threshold the resulting image $\boldsymbol{R}$ at $T_{\text {low }}$ and only consider defects that are not already contained in $D^{\text {coarse }}$ to extract the thin defect candidate map $\boldsymbol{T}$ :

$$
t_{i j}= \begin{cases}1 & \text { if } r_{i j}<T_{\text {low }} \bigwedge d_{i j}^{\text {coarse }}=0 \\ 0 & \text { otherwise }\end{cases}
$$

Note that in $\boldsymbol{T}$, large homogeneous parts are removed from the set of potential defects, but some high-frequency parts that are not defects still remain. We use $\boldsymbol{E}^{(2)}-\boldsymbol{E}^{(4)}$ to compute a defect probability map to decide which parts of $\boldsymbol{T}$ are defects. In order to compute from principal components $\boldsymbol{E}^{(k)}, k \in\{2,3,4\}$ a defect probability map, we first fit a Gaussian curve to the histograms of the eigenvectors. Let us denote the function corresponding to eigenvector $k$ as $g_{k}(x)$. To obtain the defect probability functions, we compute:

$$
f_{k}(x)=1-\frac{g_{k}(x)}{\max \left(g_{k}(x)\right)}, \text { for } k \in\{2,3,4\} \text {. }
$$

As mentioned before, the $\boldsymbol{E}^{(2)}, \boldsymbol{E}^{(3)}$, and $\boldsymbol{E}^{(4)}$ all contain partial information of the whole defect map. We compute:

$$
p_{i j}^{(k)}=f_{k}\left(e_{i j}^{(k)}\right), \text { for } k \in\{2,3,4\} .
$$

Since the principal components are orthogonal, we multiply the individual probabilities in order to get the final defect probability map $\boldsymbol{P}^{\text {defect: }}$

$$
p_{i j}^{\text {defect }}=p_{i j}^{(2)} \cdot p_{i j}^{(3)} \cdot p_{i j}^{(4)} .
$$

We use $\boldsymbol{P}^{\text {defect }}$ to reduce the false-positives of $\boldsymbol{T}$. We perform a connected component analysis (CCA) with a 4-connected neighborhood of $\boldsymbol{T}$. Let us denote $\tau_{i}, i \in\{1, \ldots, \mathrm{N}\}$ the $\mathrm{N}$ connected components resulting from the CCA. Algorithm 1 shows how the thin defect map $D^{\text {thin }}$ is computed.

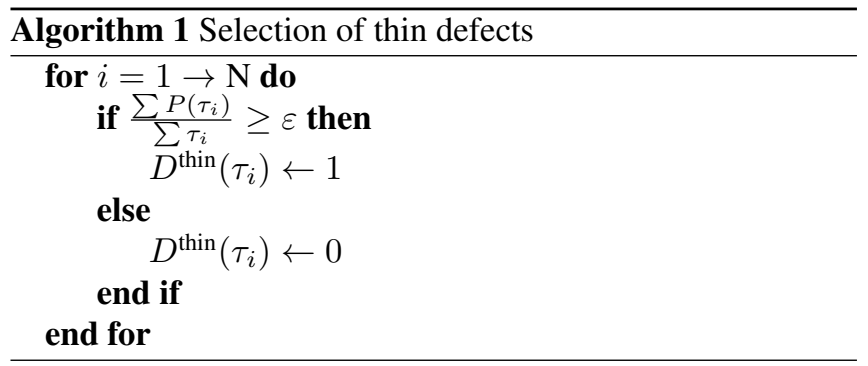




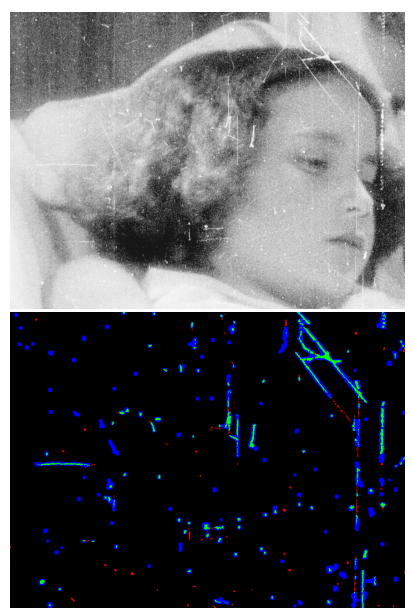

(a) $\mathrm{TP}=83.7 \%$

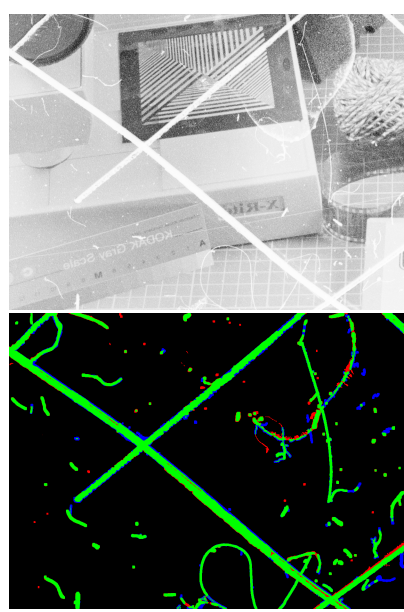

(b) $\mathrm{TP}=90.7 \%$

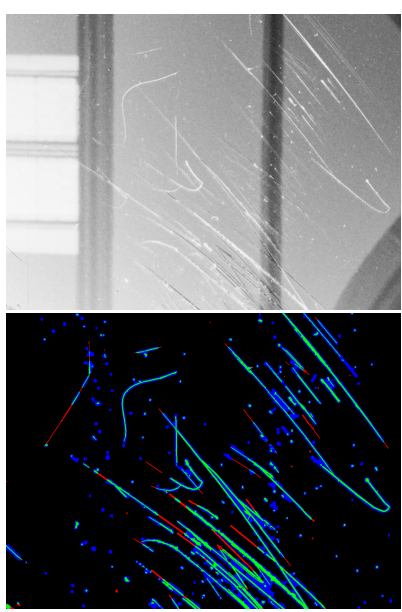

(c) $\mathrm{TP}=88.6 \%$

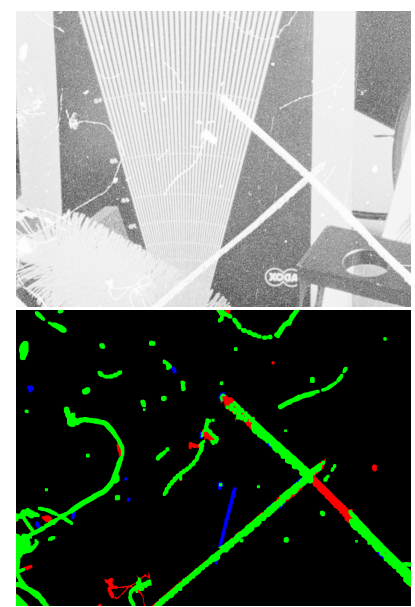

(d) $\mathrm{TP}=87.6 \%$

Fig. 4. Final defect maps of four silver halide transparencies (crops). $\mathrm{TP}=$ green, $\mathrm{FP}=$ blue, $\mathrm{FN}=$ red, $\mathrm{TN}=$ black.

$\varepsilon$ denotes a threshold which signifies the percentage of pixels that need to be in $\boldsymbol{P}^{\text {defect }}$ corresponding to a specific connected component $\tau_{i}$ so that the component is counted as defect. In our experiments, we set $\varepsilon=0.01$.

\subsection{Final Defect Map}

The last step is to combine the coarse defect map $D^{\text {coarse }}$ (see Section 3.1) with the thin defect map $D^{\text {thin }}$ explained in Section 3.2. The value of each pixel of the final defect map is computed as:

$$
d_{i j}^{\text {final }}=\max \left(d_{i j}^{\text {coarse }}+d_{i j}^{\text {thin }}, 1.0\right) .
$$

\section{RESULTS AND DISCUSSION}

We created ground truth masks for 8 direct light images containing a variety of defects. Most prominent are scratches of various sizes, as well as dust particles. We compare the output of our method with the ground truth on a pixel-by-pixel basis. Note that in general, the amount of defects is small compared to the signal. This implies that we can get a very high accuracy by simply labelling everything as not being a defect. What we are ultimately interested in is to have the highest true positive rate with as few false positives as possible. The Matthews correlation coefficient (MCC) [13] can be used as a balanced measure if the two classes have very different sizes. The value of the MCC is between $\{-1,1\}$, where the larger values indicate better prediction. Table 1

Table 1. Quantitative results (8 images)

\begin{tabular}{|l|c|c|c|c|c|c|}
\hline & TP & $\sigma_{\mathrm{TP}}$ & MCC & $\sigma_{\mathrm{MCC}}$ & Acc & $\sigma_{\text {Acc }}$ \\
\hline $\boldsymbol{D}^{\text {coarse }}$ & $59.2 \%$ & 18.6 & 0.54 & 0.23 & $95.8 \%$ & 2.9 \\
\hline $\boldsymbol{D}^{\text {final }}$ & $88.1 \%$ & 6.2 & 0.63 & 0.23 & $94.9 \%$ & 3.8 \\
\hline
\end{tabular}

shows the detected true positive rate (TP), MCC, and overall classification accuracy $\frac{T P+T N}{T P+T N+F P+F N}$ (Acc), as well as the respective standard deviations $\sigma$, for both the coarse and the final defect map. We can see that on average, there is almost a $30 \%$ improvement of detected defects from the coarse to the final defect map. While the overall accuracy is a bit lower in $D^{\text {final }}$, the MCC indicates that the overall prediction quality is better. Fig. 4 shows the results on four different film crops with various types and amounts of surface defects. We are able to detect most of the defects, while keeping the false positive rate reasonably low. Most FP surround correctly detected defects, as can be seen in Figures 4(a) and (c). As these maps are the input to subsequent restoration techniques, such as inpainting, these false positives should not interfere with performance.

Our method fails if $\boldsymbol{T}$ contains a connected component that is partly a true defect, and partly part of an edge in the film. One example of this can be seen in Fig. 4(d), where the sharp edge of the resolution target overlaps with the scratch, and hence is falsely detected as scratch. Note that since we remove everything covered by $\boldsymbol{D}^{\text {coarse }}$, the other lines of the resolution target are removed by the selection of thin defects Algorithm, as they are not connected to anything scratch related. We also tend to miss defects that are almost indistinguishable in terms of intensity values to the film signal. However, such defects are hardly visible, and might not have to be restored.

\section{CONCLUSION AND FUTURE WORK}

We present a new way of detecting dust and scratches in silver halide film by using a novel setup involving cross-polarized light and dark field illumination in a cardinal setup. We propose both a coarse and a finer defect map, and show both quantitatively and qualitatively how well our framework performs. These defect maps together with the direct light image $\boldsymbol{L}$ can then be the input to subsequent restoration algorithms. Future work includes increasing the ground truth set and investigating ways to further reduce false positives. We are also building a new acquisition setup with more light source positions, which should result in better defect probability maps. 


\section{REFERENCES}

[1] J. W. Gordon, "Dark field illumination," Journal of the Royal Microscopical Society, vol. 26, no. 2, pp. 157160, 1906.

[2] A. D. Edgar, "System and method for image recovery," US Patent 5266805, November 1993.

[3] L. F. Pascuzzi, "Wet gate embodied in a photographic strip film printer," US Patent 4082447, April 1978.

[4] T. W. Mead, "System for reducing scratch visibility," US Patent 2002/0089700, July 2002.

[5] L. Joyeux, S. Boukir, B. Besserer, and O. Buisson, "Reconstruction of degraded image sequences. application to film restoration.," Image Vision Computing, vol. 19, no. 8, pp. 503-516, 2001.

[6] R. Chang and T.K. Shih, "Spike defect detection and removal on aged films.," J. Inf. Sci. Eng., vol. 26, no. 5, pp. 1741-1753, 2010.

[7] X. Wang and M. Mirmehdi, "Archive film defect detection and removal: An automatic restoration framework," IEEE Trans. on Im. Proc., vol. 21, no. 8, pp. 3757-3769, 2012.

[8] O. Kao and J. Engehausen, "Scratch removal in digitised film sequences," in Proc. of CISST, 2000, pp. 171-179.

[9] G. Trumpy, A. Wassmer, and R. Gschwind, "Detection of dust and scratches on photographic material by combining dark field illumination and crossed polarization," 8th EOS Topical Meeting on Diffractive Optics (Presentation only), 2012.

[10] D. G. Lowe, "Distinctive image features from scaleinvariant keypoints," Int. J. Comput. Vision, vol. 60, no. 2, pp. 91-110, 2004.

[11] M. A. Fischler and R. C. Bolles, "Random sample consensus: a paradigm for model fitting with applications to image analysis and automated cartography," Commun. ACM, vol. 24, no. 6, pp. 381-395, 1981.

[12] C. Tomasi and R. Manduchi, "Bilateral Filtering for Gray and Color Images," in International Conference on Computer Vision, 1998, pp. 839-846.

[13] B.W. Matthews, "Comparison of the predicted and observed secondary structure of t4 phage lysozyme," Biochimica et Biophysica Acta (BBA) - Protein Structure, vol. 405, no. 2, pp. $442-451,1975$. 